NBI-HE-98-27

MPS-RR-1998-25

ITEP-TH-50/98

\title{
Thermodynamics of D0-branes in matrix theory ${ }^{a}$
}

\author{
J. Ambjørn \\ Niels Bohr Institute \\ Blegdamsvej 17 \\ Copenhagen, 2100 Denmark \\ ambjorn@nbi.dk \\ Y. M. Makeenko \\ Institute of Theoretical and Experimental Physics \\ B. Cheremushkinskaya 25 \\ Moscow, 117218 Russian Federation \\ makeenko@itep.ru \\ G. W. Semenoff \\ Department of Physics and Astronomy \\ University of British Columbia \\ 6224 Agricultural Road \\ Vancouver, British Columbia V6T 1Z1, Canada \\ semenoff@physics.ubc.ca
}

\begin{abstract}
We examine the matrix theory representation of D0-brane dynamics at finite temperature. In this case, violation of supersymmetry by temperature leads to a non-trivial static potential between D0-branes at any finite temperature. We compute the static potential in the 1loop approximation and show that it is short-ranged and attractive. We compare the result with the computations in superstring theory. We show that thermal states of D0-branes can be reproduced by matrix theory only when certain care is taken in integration over the moduli space of classical solutions in compactified time.
\end{abstract}

${ }^{a}$ This work is supported in part by NATO Grant CRG 970561, by NSERC and by MaPhySto - Centre for Mathematical Physics and Stochastics. 


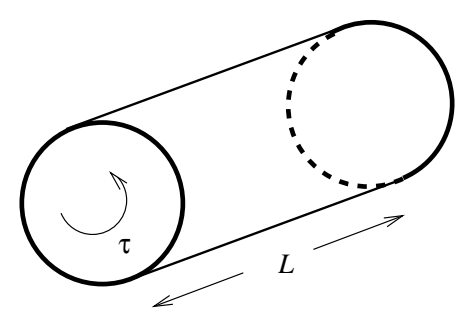

Figure 1: Annulus diagram for D0-brane interactions. The bold lines represent world-lines of the D0-branes, separated by the distance $L$, which go along the periodic temporal direction. They bound the string worldsheet.

\section{Introduction}

Dirichlet p-branes are p+1-dimensional hypersurfaces on which superstrings can begin and end (see阳 for a review). The low energy dynamics of an ensemble of N parallel Dp-branes can be described by the $\mathrm{U}(\mathrm{N})$ supersymmetric gauge theory obtained by dimensional reduction of ten dimensional supersymmetric Yang-Mills theory to the p+1-dimensional world-volume

of the brane $e^{3}$ The Yang-Mills theory gives an accurate perturbative representation of the Dp-brane dynamics when the separations between the branes is larget truncation of the full string spectrum to the lowest energy modes. The full string theoretical interactions between a pair of Dp-branes is computed by considering the annulus diagram shown in fig. 1. The short distance asymptotics of this diagram are dominated by the open string sector whose lowest modes are the fields of ten dimensional supersymmetric Yang-Mills theory. On the other hand, long distance asymptotics are most conveniently described by the dual description of this diagram as a closed string exchange and the relevant field theoretical modes are those of ten dimensional supergravity. That these are also represented by the dimensionally reduced super Yang-Mills theory is a result of supersymmetry and the fact that, for fixed Dp-brane positions, the ground state is a BPS state. At zero temperature, because of supersymmetry, the interaction potential between a pair of static D0-branes vanishes independently of their separation. Their effective action has been computed in an expansion in their velocities, divided by powers of the separation and is known to be日

$$
S_{\mathrm{eff}}(T=0)=\int d t\left(\frac{1}{2 g_{s} \sqrt{\alpha^{\prime}}} \sum_{\alpha=1}^{2}\left(\dot{\vec{q}}^{\alpha}\right)^{2}-\frac{15}{16}\left(\alpha^{\prime}\right)^{3} \frac{\left|\dot{\vec{q}}^{1}-\dot{\vec{q}}^{2}\right|^{4}}{\left|\vec{q}^{1}-\vec{q}^{2}\right|^{7}}+\ldots\right)
$$

This result agrees with the effective potential for the interaction of D0-branes in ten dimensional supergravity. Note that, for weak string coupling, the D0-brane is very heavy.

In this paper, we shall consider the description of D0-brane interactions in type IIA superstring theory using matrices. Even at very low temperatures, non-BPS states are important to the leading temperature dependence. We perform 1-loop computation of the effective interaction between static D0-branes in the matrix theory at finite temperature and compare with the known superstring computations. We show that the results of the two computa- 
tions are similar in the low temperature limit but an extra integration over the temporal component of the gauge field, is present in the matrix theory. At finite temperature, because the Euclidean time is compact, the temporal gauge field can not be removed by a gauge transformation. This integration is needed in order to describe correctly thermodynamics of D0-branes both in the matrix and superstring theories.

The paper is organized as follows. In section 2 we discuss the formulation of the matrix theory at finite temperature. In section 3 we perform one loop computation of the effective interaction between static D0-branes at finite temperature and show that it is attractive, and short-ranged. In section 4 we compare this result with the superstring computations and discuss the conditions under which the two computations agree. Section 5 is devoted to the discussion of our results and, in particular, the origin of the divergence of the classical thermal partition function of D0-branes which is cured by quantum statistics.

\section{Matrix theory at finite temperature}

We shall consider the matrix theory description 8 of the effective dynamics of D0-branes in a type-IIA superstring theory which is derived by the reduction of ten dimensional supersymmertic Yang-Mills theory which has the action

$$
S_{\mathrm{YM}}[A, \theta]=\frac{1}{g_{Y M}^{2}} \int d \tau \operatorname{TR}\left(\frac{1}{4} F_{\mu \nu}^{2}+\frac{i}{2} \theta \gamma_{\mu} D_{\mu} \theta\right)
$$

to zero spatial dimension: $A_{\mu}=A_{\mu}(\tau), \theta=\theta(\tau)$.

The thermal partition function of this theory is given by

$$
Z_{\mathrm{YM}}=\int[d A(\tau)][d \theta(\tau)] \exp \left(-S_{\mathrm{YM}}[A, \theta]\right)
$$

where $S_{\mathrm{YM}}$ is the Euclidean action and the time coordinate is periodic. The bosonic and fermionic coordinates have periodic and anti-periodic boundary conditions,

$$
\begin{array}{r}
A_{\mu}(\tau+\beta)=A_{\mu}(\tau), \\
\theta(\tau+\beta)=-\theta(\tau), \\
\beta=1 / k_{B} T,
\end{array}
$$

where $T$ is the temperature and $k_{B}$ is Boltzmann's constant. Gauge fixing will be necessary and will involve introducing ghost fields which will have periodic boundary conditions.

The representation (3) of the thermal partition function can be derived in the standard way starting from the known Hamiltonian of the matrix theory $\mathrm{G}$ and representing the thermal partition function

$$
Z_{\mathrm{YM}}=\operatorname{tr} e^{-\beta H}
$$


via the path integral. The trace is calculated here over all states obeying Gauss's law which is taken care by the integration over $A_{0}$ in (3). This representation of the matrix theory at finite temperature have been already discussed 9.10 .11 , but the temperature induced interaction between $D 0$-branes described below was never identified.

In matrix theory, the diagonal components of the gauge fields, $\vec{a}^{\alpha} \equiv \vec{A}^{\alpha \alpha}$, are interpreted as the position coordinates of the $\alpha$-th D0-brane and they should be treated as collective variables. Static configurations play a special role since they satisfy classical equations of motion with the periodic boundary conditions and dominate the path integral as $g_{\mathrm{YM}}^{2} \rightarrow 0$. Notice that there are no such static zero modes for fermionic components since they would not satisfy the antiperiodic boundary conditions. 9 This is an important difference from the zero temperature case and a manifestation of the fact that supersymmetry is explicitly broken at non-zero temperature.

In the following, we will construct an effective action for these coordinates by integrating the off-diagonal gauge fields, the fermionic variables and the ghosts,

$$
S_{\mathrm{eff}}\left[\vec{a}^{\alpha}\right] \equiv-\ln \int\left[d a_{0}^{\alpha}\right] \prod_{\alpha \neq \beta}\left[d A_{\mu}^{\alpha \beta}\right][d \theta][d \mathrm{ghost}] \exp \left(-S_{\mathrm{YM}}-S_{\mathrm{gf}}-S_{\mathrm{gh}}\right)
$$

Generally, this integration can only be done in the a simultaneous loop expansion and expansion in the number of derivatives of the coordinates $\vec{a}^{\alpha}$. Such an expansion is accurate in the limit where $\left|\vec{a}^{\alpha}-\vec{a}^{\beta}\right|$ are large for each pair of D0-branes and where the velocities are small. Since these variables are periodic in Euclidean time, small velocities are only possible at low temperatures. The remaining dynamical problem then defines the statistical mechanics of a gas of D0-branes,

$$
Z_{\mathrm{YM}}=\int \prod_{\tau, \alpha}\left[d \vec{a}^{\alpha}(\tau)\right] \exp \left(-S_{\mathrm{eff}}\left[\vec{a}^{\alpha}\right]\right)
$$

We expect that the zero temperature limit of $S_{\text {eff }}$ reduces to (1).

We shall find several subtleties with this formulation. If the effective D0-brane action is to reproduce the results of a string theoretical computation, the integration over $a_{0}^{\alpha}$ must be performed in both cases.

The effective action is a symmetric functional of the position variables $\vec{a}^{\alpha}(\tau)$. Only the configuration of these coordinates needs to be periodic. Therefore the individual position should be periodic up to a permutation. The variables in the path integral (9) should therefore be periodic up to a permutation and the integral should be summed over the permutations.

\footnotetext{
${ }^{b}$ This is a difference between our computation at finite temperature and computations of the Witten index for the matrix theory where fermions obey periodic boundary conditions.
} 


\section{One loop computation}

We will compute the effective action $S_{\text {eff }}$ in a simultaneous expansion in the number of loops and in powers of time derivatives of the D0-brane positions.

We decompose the gauge field into diagonal and off-diagonal parts,

$$
A_{\mu}^{\alpha \beta}=a_{\mu}^{\alpha} \delta^{\alpha \beta}+g_{\mathrm{YM}} \bar{A}_{\mu}^{\alpha \beta}
$$

where $\bar{A}_{\mu}^{\alpha \alpha}=0$ so that the curvature is

$$
F_{\mu \nu}^{\alpha \beta}=\delta^{\alpha \beta} f_{\mu \nu}^{\alpha}+g_{\mathrm{YM}} D_{\mu}^{\alpha \beta} \bar{A}_{\nu}^{\alpha \beta}-g_{\mathrm{YM}} D_{\nu}^{\alpha \beta} \bar{A}_{\mu}^{\alpha \beta}-i g_{\mathrm{YM}}^{2}\left[\bar{A}_{\mu}, \bar{A}_{\nu}\right]^{\alpha \beta}
$$

where

$$
f_{\mu \nu}^{\alpha}=\partial_{\mu} a_{\nu}^{\alpha}-\partial_{\nu} a_{\mu}^{\alpha}
$$

and

$$
D_{\mu}^{\alpha \beta}=\partial_{\mu}-i\left(a_{\mu}^{\alpha}-a_{\mu}^{\beta}\right) .
$$

In the Yang-Mills term in the action, we keep all orders of the diagonal parts of the gauge field and expand up to second order in the off-diagonal components,

$\frac{1}{g_{\mathrm{YM}}^{2}} \operatorname{TR}\left(F_{\mu \nu}^{2}\right)=\sum_{\alpha} \frac{1}{g_{\mathrm{YM}}^{2}}\left(f_{\mu \nu}^{\alpha}\right)^{2}+2 \sum_{\alpha \beta} \bar{A}_{\mu}^{\beta \alpha}\left(\delta_{\mu \nu} \overleftarrow{D}_{\lambda}^{\beta \alpha} \vec{D}_{\lambda}^{\alpha \beta}-\overleftarrow{D}_{\mu}^{\beta \alpha} \vec{D}_{\nu}^{\alpha \beta}+2 i\left(f_{\mu \nu}^{\alpha}-f_{\mu \nu}^{\beta}\right)\right) \bar{A}_{\nu}^{\alpha \beta}+\ldots$

We will fix the gauge

$$
D_{\mu}^{\alpha \beta} \bar{A}_{\mu}^{\alpha \beta}=0
$$

This entails adding the Fadeev-Popov ghost term to the action

$$
S_{\mathrm{gh}}=\int \sum_{\alpha \beta}\left\{\bar{c}^{\alpha \beta}\left(-D_{\mu}^{\alpha \beta}\right)^{2} c^{\beta \alpha}+i g_{\mathrm{YM}} \bar{c}^{\beta \alpha} D_{\mu}^{\alpha \beta}\left[\bar{A}_{\mu}, c\right]\right\}
$$

There is a residual gauge invariance under the abelian transformation,

$$
\begin{array}{r}
\bar{A}_{\mu}^{\alpha \beta} \rightarrow \bar{A}_{\mu}^{\alpha \beta} e^{i\left(\chi^{\alpha}-\chi^{\beta}\right)}, \\
a_{\mu}^{\alpha} \rightarrow a_{\mu}^{\alpha}+\partial_{\mu} \chi^{\alpha} .
\end{array}
$$

We shall use this gauge freedom to set the additional condition

$$
\partial_{0} a_{0}^{\alpha}=0
$$

and to fix the constant ${ }^{c}$

$$
-\pi / \beta<a_{0}^{\alpha} \leq \pi / \beta
$$

${ }^{c}$ These constants are related to the eigenvalues of the holonomy

$$
\mathrm{P} \exp \left(i \int_{0}^{\beta} d \tau A_{0}(\tau)\right)=\Omega^{\dagger} \operatorname{diag}\left(e^{i \beta a_{0}^{1}}, \ldots, e^{i \beta a_{0}^{\mathrm{N}}}\right) \Omega
$$

known as the Polyakov loop winding along the compact Euclidean time. It can not be made trivial by the gauge transformation if $T \neq 0$. 
The ghost for this gauge fixing condition decouples.

Keeping terms up to quadratic order in $\bar{A}, c, \bar{c}, \theta$, the action is

$$
\begin{array}{r}
S=\int\left\{\frac{1}{4 g_{\mathrm{YM}}^{2}} \sum_{\alpha}\left(f_{\mu \nu}^{\alpha}\right)^{2}+\frac{1}{2} \sum_{\alpha \beta} \bar{A}_{\mu}^{\beta \alpha}\left(-\delta_{\mu \nu} D_{\lambda}^{\beta \alpha} D_{\lambda}^{\alpha \beta}+D_{\mu}^{\beta \alpha} D_{\nu}^{\alpha \beta}+2 i\left(f_{\mu \nu}^{\alpha}-f_{\mu \nu}^{\beta}\right)\right) \bar{A}_{\nu}^{\alpha \beta}\right. \\
\left.+\sum_{\alpha \beta} \bar{c}^{\beta \alpha}\left(D_{\mu}^{\alpha \beta}\right)^{2} c^{\alpha \beta}+\frac{i}{2} \theta^{\beta \alpha} \gamma_{\mu} D_{\mu}^{\alpha \beta} \theta^{\alpha \beta}\right\} .
\end{array}
$$

The effective action obtained by integrating over $\bar{A}, \bar{c}, c, \theta$ is

$$
\begin{array}{r}
S_{\text {eff }}=\int \sum_{\alpha} \frac{1}{4 g_{\mathrm{YM}}^{2}}\left(f_{\mu \nu}^{\alpha}\right)^{2}+\sum_{\alpha \neq \beta}\left\{\frac{1}{2} \mathrm{TR} \ln \left(-\delta_{\mu \nu}\left(D_{\mu}^{\alpha \beta}\right)^{2}+2 i\left(f_{\mu \nu}^{\alpha}-f_{\mu \nu}^{\beta}\right)\right)\right. \\
\left.-\mathrm{TR} \ln \left(-\left(D_{\mu}^{\alpha \beta}\right)^{2}\right)-\frac{1}{2} \mathrm{TR} \ln \left(i \gamma_{\mu} D_{\mu}^{\alpha \beta}\right)\right\} .
\end{array}
$$

\subsection{Leading order in time derivatives}

We will evaluate the determinants on the right-hand-side of (21) in an expansion in powers of the derivatives of $\vec{a}(\tau)$. The leading order term can be found by setting $\vec{a}=$ const.. In this case, $f_{\mu \nu}^{\alpha}=0$ and (here we retain the tree-level term with time derivatives)

$$
S_{\text {eff }}=8 \sum_{\alpha<\beta}\left\{\mathrm{TR}_{B} \ln \left(-\left(D_{\mu}^{\alpha \beta}\right)^{2}\right)-\mathrm{TR}_{F} \ln \left(-\left(D_{\mu}^{\alpha \beta}\right)^{2}\right)\right\}
$$

where the subscript $B$ denotes contributions from the gauge fields and ghosts, whereas $F$ denotes those from the adjoint fermions. The determinants should be evaluated with periodic boundary conditions for bosons and anti-periodic boundary conditions for fermions. (Note that, because of supersymmetry, if both bosons and fermions had identical boundary conditions the determinants would cancel. This would give the well-known result that the lowest energy state is a BPS state whose energy does not depend on the relative separation of the D0-branes.) The boundary conditions are taken into account by introducing Matsubara frequencies, so that

$$
e^{-S_{\mathrm{eff}}}=e^{-S_{0}} \beta^{\mathrm{N}} \int \frac{d a_{0}^{\alpha}}{2 \pi} \prod_{\alpha<\beta} \prod_{n=-\infty}^{\infty}\left(\frac{\left(\frac{2 \pi n}{\beta}+\frac{\pi}{\beta}+a_{0}^{\alpha}-a_{0}^{\beta}\right)^{2}+\left|\vec{a}_{\alpha}-\vec{a}_{\beta}\right|^{2}}{\left(\frac{2 \pi n}{\beta}+a_{0}^{\alpha}-a_{0}^{\beta}\right)^{2}+\left|\vec{a}_{\alpha}-\vec{a}_{\beta}\right|^{2}}\right)^{8}
$$

Using the formula

$$
\prod_{n=-\infty}^{\infty}\left(\frac{2 \pi n}{\beta}+\omega\right)=\sin \left(\frac{\beta \omega}{2}\right)
$$

we obtain the result

$$
e^{-S_{\mathrm{eff}}}=e^{-S_{0}} \beta^{\mathrm{N}} \int \frac{d a_{0}^{\alpha}}{2 \pi} \prod_{\alpha<\beta}\left(\frac{\cosh \beta\left|\vec{a}^{\alpha}-\vec{a}^{\beta}\right|+\cos \beta\left(a_{0}^{\alpha}-a_{0}^{\beta}\right)}{\cosh \beta\left|\vec{a}^{\alpha}-\vec{a}^{\beta}\right|-\cos \beta\left(a_{0}^{\alpha}-a_{0}^{\beta}\right)}\right)^{8}
$$


In order to find the effective action for $\vec{a}^{\alpha}$, it is now necessary to integrate the temporal gauge fields $a_{0}^{\alpha}$ over the domain $(-\pi / \beta, \pi / \beta]$. This integration implements the projection onto the gauge invariant eigenstates of the matrix theory Hamiltonian.

In the case where there is a single pair of D0-branes, $\mathrm{N}=2$, the integration over $a_{0}^{\alpha}$ in (25) can be done explicitly to obtain the effective action

$$
S_{\mathrm{eff}}=\int_{0}^{\beta} d \tau\left\{\sum_{1}^{2} \frac{\left(\dot{\vec{a}}^{\alpha}\right)^{2}}{2 g_{\mathrm{YM}}^{2}}-\frac{1}{\beta} \ln \left(\frac{P(z)}{\left(1-z^{2}\right)^{15}}\right)\right\}
$$

where

$$
\begin{aligned}
P(z)=1+ & 241 z^{2}+12649 z^{4}+254009 z^{6}+2434901 z^{8}+12456773 z^{10}+36119181 z^{12} \\
+61178589 z^{14}+6191459 z^{16}+ & 36109171 z^{18}+12462779 z^{20}+2432171 z^{22} \\
+ & 254919 z^{24}+12439 z^{26}+271 z^{28}-z^{30}
\end{aligned}
$$

$z=\exp \left(-\beta\left|\vec{a}^{1}-\vec{a}^{2}\right|\right)$ and we have included the tree level term, which gives the non-relativistic kinetic energies of the D0-branes.

The effective action has the low temperature expansion

$$
\begin{array}{r}
S_{\mathrm{eff}}=\int_{0}^{\beta} d \tau\left(\frac{1}{2 g_{\mathrm{YM}}^{2}} \sum_{\alpha}\left(\dot{\vec{a}}^{\alpha}\right)^{2}-\frac{1}{\beta}\left(256 e^{-2 \beta\left|\vec{a}^{1}-\vec{a}^{2}\right|}-16384 e^{-4 \beta\left|\vec{a}^{1}-a^{2}\right|}\right.\right. \\
\left.\left.+\frac{5614336}{3} e^{-6 \beta\left|\vec{a}^{1}-\vec{a}^{2}\right|}+\ldots\right)\right)
\end{array}
$$

We shall compare in the following section this result with the superstring computation of the effective interaction between D0-branes.

\section{String theoretical interactions}

The effective interactions of D0-branes in superstring theory is given by computing the annulus diagram shown in fig. 11. This was done in ref. 12 (and in 13 for Dp-branes). The result of summing over physical (GSO projected) superstring states gives the free energy

$$
F[L, \beta, \nu]=\frac{8}{\sqrt{\pi \alpha^{\prime}}} \int_{0}^{\infty} \frac{d l}{l^{3 / 2}} e^{-L^{2} l / 4 \pi^{2} \alpha^{\prime}} \Theta_{2}\left(\nu \mid \frac{i \beta^{2}}{\pi \alpha^{\prime} l}\right) \prod_{n=1}^{\infty}\left(\frac{1+e^{-n l}}{1-e^{-n l}}\right)^{8}
$$

where

$$
\Theta_{2}(\nu \mid i z)=\sum_{q=-\infty}^{\infty} e^{-\pi z(2 q+1)^{2} / 4+i \pi(2 q+1) \nu},
$$

$L$ is the brane separation and $\nu$ is a parameter which weights the winding numbers of strings around the periodic time direction. An extra factor of 2 accounting for the exchange of the two ends of the superstring 1 ending on each of the two D0-branes is inserted in (29). 
The product in the integrand represents the sum over string states, with requisite degeneracies,

$$
8 \prod_{n=1}^{\infty}\left(\frac{1+e^{-n l}}{1-e^{-n l}}\right)^{8}=\sum_{N=0}^{\infty} d_{N} e^{-N l}
$$

where $d_{N}$ is the degeneracy of the either superstring state at level $N$. For the lowest few levels, $d_{0}=8$ and $E_{0}=L / 2 \pi \alpha^{\prime}$.

Inserting (31) in (29) and integrating over $l$, the free energy has the form

$$
F(\beta, L, \nu)=\frac{2}{\beta} \sum_{N=0}^{\infty} d_{N} \ln \left|\frac{1-e^{-\beta E_{N}+i \pi \nu}}{1+e^{-\beta E_{N}+i \pi \nu}}\right|
$$

where the string energies are given by the formula

$$
E_{N}=\frac{L}{2 \pi \alpha^{\prime}} \sqrt{1+\frac{4 \pi^{2} \alpha^{\prime} N}{L^{2}}} .
$$

This results in the partition function

$$
Z_{\mathrm{str}}(\beta, L, \nu) \equiv e^{-\beta F}=\prod_{N=0}^{\infty}\left|\frac{1+e^{-\beta E_{N}+i \pi \nu}}{1-e^{-\beta E_{N}+i \pi \nu}}\right|^{2 d_{N}} .
$$

The physical meaning of the last formula is obvious: the partition function equals the ratio of the Fermi and Bose distributions with the power being twice the degeneracy of states and $i \nu$ playing the role of a chemical potential. The factor of 2 in the exponent $2 d_{N}$ in (34) is due to the interchange of the superstring ends as is already mentioned. It will provide the agreement with the matrix theory computation.

In order to compare with the Yang-Mills computation, we should first re-scale the coor-

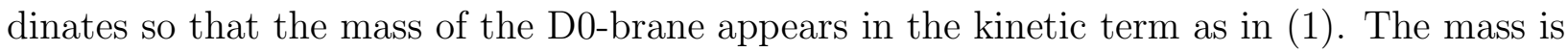
given by the formula

$$
M=\frac{1}{g_{s} \sqrt{\alpha^{\prime}}}
$$

and the Yang-Mills coupling $g_{\mathrm{YM}}$ is related to the string coupling $g_{s}$ by the equation

$$
g_{\mathrm{YM}}^{2}=\frac{g_{s}}{4 \pi^{2}\left(\alpha^{\prime}\right)^{3 / 2}} .
$$

The physical coordinate of the $\alpha$-th D0-brane is identified with

$$
\vec{q}^{\alpha}=2 \pi \alpha^{\prime} \vec{a}^{\alpha} .
$$

Taking $\mathrm{N}=2$ in (25) and identifying $L=2 \pi \alpha^{\prime}\left|\vec{a}^{1}-\vec{a}^{2}\right|$, we see that the integrand in (25) coincides with (34) truncated to the massless modes $(N=0)$ provided $\nu=\beta\left(a_{0}^{1}-a_{0}^{2}\right)$. 
It is clear that the integral over $a_{0}^{\alpha}$ is responsible for the "mismatch" of the effective actions between the string theory computation and matrix theory computation of the free energy. In the string theory done in the spirit of ref. 12 , the parameter $\nu$ appears in the same place as $\beta\left(a_{0}^{1}-a_{0}^{2}\right) / \pi$ but is not integrated. It is associated with the interaction of the ends of the open string with an Abelian gauge field background $A_{\mu}(\tau, \vec{x})$ :

$$
S_{\text {int }}=\int d x^{\mu} A_{\mu}
$$

If the ends are separated by the distance $L$, e.g. along the first spatial axis, then

$$
\nu=\int_{0}^{\beta} d \tau\left(A_{0}(\tau, 0, \ldots)-A_{0}(\tau, L, \ldots)\right)
$$

since $\dot{x}_{\mu}(\tau)=(1, \overrightarrow{0})$ on the boundaries. The matrix theory automatically takes into account the integration over the background field while in the string theory calculation of ref. 12 the background field is fixed. This is just a reflection of the fact that matrix theory is an effective low-energy theory of $D$-branes, while the older string theory did not treat the boundaries as dynamical objects. However, it is interesting to notice how close some of the earlier string papers came to such a description simply by the requirement of consistency 14 . Further, in the context of matrix theory it is natural to take the exponential of the free energy (32) as in eq. (34), and only integrete over $\nu$ afterwards, a procedure not entirely obvious in a string theory where the boundaries are not dynamical objects. This will be discussed further in the next section.

An exact coincidence between the matrix theory and superstring results is possible only when the higher stringy modes are suppressed. Usually, the truncation of the string spectrum to get Yang-Mills theory is valid for small $\alpha^{\prime}$, that is when we are interested in temperatures which are much smaller than $1 / \sqrt{\alpha^{\prime}}$. In fact, the condition in our case is a little different than this once the length $L$ appears as a parameter in the spectrum (33). Then, the spectrum can be truncated at the first level only when

$$
\frac{1}{\beta} \equiv k_{B} T \ll \sqrt{\left(\frac{L}{2 \pi \alpha^{\prime}}\right)^{2}+\frac{1}{\alpha^{\prime}}}-\frac{L}{2 \pi \alpha^{\prime}}
$$

which is the energy gap between the first two levels. If the temperature is small, this condition is always satisfied unless the length $L$ is not too large. In other words the truncation of the spectrum to the lightest modes is valid for $\beta \gg L$ (or $T L \ll 1$ ).

It is also interesting to discuss what happens in the opposite limit $L \gg \beta$ where the interaction between D0-branes is mediated by the lightest closed string modes. The superstring free energy can be evaluated in this limit by the standard modular transformation which relates the annulus diagram for an open string with a cylinder diagram for a closed 
string. Introducing the new integration variable $s=2 \pi^{2} / l$, we rewrite (29) as 12

$$
F[L, \beta, \nu]=\frac{8 \pi^{4}}{\sqrt{2 \pi \alpha^{\prime}}} \int_{0}^{\infty} \frac{d s}{s^{9 / 2}} e^{s} e^{-L^{2} / 2 s \alpha^{\prime}} \Theta_{2}\left(\nu \mid \frac{i \beta^{2} s}{2 \pi^{3} \alpha^{\prime}}\right) \prod_{n=1}^{\infty}\left(\frac{1-e^{-(2 n+1) s}}{1-e^{-2 n s}}\right)^{8} .
$$

In the limit where the brane separation is large the integration over $s$ is concentrated in the region of large $s \sim L^{2}$. Substituting the large- $z$ asymptotics

$$
\Theta_{2}(\nu \mid i z) \rightarrow 2 \cos (\pi \nu) e^{-\pi z / 4}
$$

and evaluating the saddle-point integral, we get

$$
F[L, \beta, \nu] \propto \cos (\pi \nu) \frac{\left(\beta^{2}-8 \pi^{2} \alpha^{\prime}\right)^{3 / 2}}{L^{4}} e^{-L \sqrt{\beta^{2}-8 \pi^{2} \alpha^{\prime}} / 2 \pi \alpha^{\prime}} .
$$

Exponentiating and integrating over $\nu$, we have

$$
\int_{-1}^{1} d \nu Z_{\mathrm{str}}(\beta, L, \nu) \propto \frac{\beta^{2}\left(\beta^{2}-8 \pi^{2} \alpha^{\prime}\right)^{3}}{L^{8}} e^{-L \sqrt{\beta^{2}-8 \pi^{2} \alpha^{\prime}} / \pi \alpha^{\prime}} .
$$

Taking into account (37) the exponent at the low temperatures is the same as in (28) but the pre-exponential differs. The dependence of the pre-exponential on $L$ in the superstring case emerges because the splitting between energy states in (33) is of order $1 / L$ and the truncation condition (40) is no longer satisfied when $L$ is large. Higher stringy modes are then not separated by a gap and the continuum spectrum results in the $L$-dependence of the preexponential. As usual, the limits of $L \rightarrow \infty$ and $T \rightarrow 0$ are not interchangeable in the superstring theory.

\section{Discussion}

Our main results concern D0-brane dynamics at finite temperatures. We have computed the 1-loop effective action for the interaction of static D0-branes in the matrix theory at finite temperature and compared it with the analogous superstring computation. We have seen that an extra integration over the eigenvalues of the holonomy along the compactified Euclidean time is present in the matrix theory. The two computations agrees in the low temperature limit provided the superstring thermal partition function is integrated over the Abelian gauge fields $a_{0}$ 's living on D0-branes.

The integration over $a_{0}$ 's is of course natural in the context of the Yang-Mills theory, where it expresses that only gauge-invariant states should contribute to $\mathrm{TR} e^{-\beta H}$. But it is also natural from the point of view of the D0-brane physics. It can be seen as follows. Suppose we make a T-duality transformation, which interchanges the Neumann and Dirichlet boundary conditions, along the compactified Euclidean time direction. Then $a_{0}$ 's become the 
coordinates of D-instantons on the dual circle. The integration over $a_{0}$ 's becomes now the integration over the positions of D-instantons. The partition function should involve such an integration over the collective coordinates and since they are collective coordinates the integration appears in front of the exponential of the effective action, not in the action itself. Viewed in terms of D0-branes and open strings, we have a gas of D0-branes with open strings between them. The individual strings might have a winding number $q$ (more precisely $2 q+1$ in the case of superstrings), describing the winding around the finite-temperature space-time cylinder. The energy of such states are $\propto \beta q / 2 \pi \alpha^{\prime}$. However, the $q$ 's satisfy $\sum q=0$ as a result of the integration over $a_{0}$. Physically this constraint is most easily understood by going to the closed string channel where we have closed string boundary state on the dual circle with radius $\tilde{\beta}=4 \pi^{2} \alpha^{\prime} / \beta$ localized at the point $(\nu \tilde{\beta}, \vec{q})$. Passing to the momentum representation, we write

$$
|B, \vec{q}, \nu\rangle=\sum_{q=-\infty}^{\infty} e^{-2 i \pi \nu q}\left|B, \vec{q}, p_{0}=2 \pi q / \tilde{\beta}\right\rangle .
$$

Here the temporal momentum is quantized as $p_{0}=2 \pi q / \tilde{\beta}=q \beta / 2 \pi \alpha^{\prime}$, which lead to the same energy as the above mentioned open string states. In this representation $\sum q=0$ simply expresses momentum conservation in the thermal direction.

The effective static potential between two D0-branes emerges because supersymmetry is broken by finite temperature. This effect of breaking supersymmetry is somewhat analogous to the velocity effects at zero temperature where the matrix theory and superstring computations agree to the leading order of the velocity expansion 6 . We have thus shown that the leading term in a low temperature expansion is correctly reproduced by the matrix theory. The discrepancy between the matrix theory and superstring computations, which we have observed in the limit of large distances $L T \gg 1$, does not contradict this statement since temperature the limits of large distances and small temperatures are not interchangeable.

An interesting feature of the effective static potential between D0-branes is that it is logarithmic and attractive at short distances. In the matrix theory, the singularity of the computed 1-loop potential occurs when the distance between the D0-branes vanishes and the $\mathrm{SU}(\mathrm{N})$ symmetry which is broken by finite distances is restored. The integration over the off-diagonal components can no longer be treated in the 1-loop approximation! In the superstring theory, the singularity is exactly the same as in the matrix theory since it is determined only by the massless bosonic modes (the NS sector in the superstring theory). Its origin is not due to the presence of massless photon states in the spectrum. Putting $\nu_{1}=\nu_{2}$ in the above D-instanton picture on the dual temporal circle, we see that the mass of the lowest states, associated with the winding numbers $2 q+1= \pm 1$ is $\tilde{\beta} / 2 \pi \alpha^{\prime}$. The divergence at $L=0$ emerges, in this picture, after summing over all the open string states 
since no single state has such a divergence. It shows up only at finite temperature where the winding number $q$ exists.

It is important to notice that the computed partition functions take into account only thermal fluctuations of superstring stretched between D0-branes but not the fluctuations of D0-branes themselves. To calculate the thermal partition function of D0-branes, a further path integration over their periodic trajectories $\vec{a}(\tau)$ is to be performed as in (9). One might think that classical statistics is applicable to this problem since the D0-branes are very heavy as $g_{\mathrm{YM}}^{2} \rightarrow 0$ so that one could restrict himself by the static approximation. This is however not the case due to the singularity of the effective static potential at small distances. The integral over the D0-brane positions $\vec{a}$ 's is divergent when the two positions coincide.

However, this singularity is only in the classical partition function. The path integral over the periodic trajectories $\vec{a}(\tau)$ that we actually have to do can not diverge since the 2-body quantum mechanical problem has a well-defined spectrum. The path integral can then be evaluated as $\sum_{n} \exp \left(-\beta E_{n}\right)$ where $E_{n}$ are in the spectrum of the operator $H=P^{2} / M+V_{\text {eff }}$. There certainly should not be the bound state energy eigenvalue at negative infinity for this quantum mechanical problem which implies the convergence of the path integral. These issues which are related to thermodynamics of D0-branes will be considered in a separate publication.

Let us finally discuss when the 1-loop appoximation that we have done is applicable. The loop expansion in Yang-Mills theory computation is valid only in the limit where

$$
g_{\mathrm{YM}}^{2} /|\vec{a}|^{3} \sim g_{s}\left(\frac{\sqrt{\alpha^{\prime}}}{L}\right)^{3}
$$

is small. This is due to the fact that the distance $L$ plays the role of a Higgs mass which cuts off the infrared divergences of the loop expansion in the $0+1$-dimensional gauge theory. Thus, the perturbative Yang-Mills theory computation is good when

$$
g_{s}^{1 / 3} \sqrt{\alpha^{\prime}} \ll L
$$

This can be satisfied if either the string coupling is small or if the D0-brane separation is large compared with the string length scale. In the latter case, the truncation of the spectrum to the lightest modes is still valid when

$$
k_{B} T \ll \frac{1}{L} \ll \frac{1}{g_{s}^{1 / 3} \sqrt{\alpha^{\prime}}}
$$

Note that the first inequality which is independent of both the string scale and the string coupling is the one already discussed in the previous section. In this case, the temperature must be less than the inverse distance between D0-branes. In the case where the string 
coupling $g_{s}$ is small, the criterion for validity of truncation of the spectrum becomes

$$
k_{B} T<1 / \sqrt{\alpha^{\prime}}
$$

that is the usual one.

\section{Acknowlegments}

We are gratefull to P. Di Vecchia, G. Ferretti, A. Gorsky, M. Green, M. Krogh, N. Nekrasov, N. Ohta, P. Olesen, L. Thorlacius and K. Zarembo for very useful discussions. J.A. and Y.M. acknowledge the support from MaPhySto financed by the Danish National Research Foundation and from INTAS under the grant $96-0524$. The work by Y.M. is supported in part by the grants CRDF 96-RP1-253 and RFFI 97-02-17927.

1. J. Polchinski, hep-th/9611050.

2. W. Taylor, hep-th/9801182.

3. E. Witten, Nucl. Phys. B460 (1996) 335, hep-th/9510135.

4. U. Danielsson, G. Ferretti and B. Sundborg, Int. J. Mod. Phys. A11 (1996) 5463, hep-th/9603081.

5. D. Kabat and P. Pouliot, Phys. Rev. Lett. 77 (1996) 1004, hep-th/9603127.

6. M. R. Douglas, D. Kabat, P. Pouliot and S. Shenker, Nucl. Phys.. B485 (1997) 85, hep-th/9608024.

7. C. Bachas, Phys. Lett. B374 (1996) 37, hep-th/9511043.

8. T. Banks, W. Fischler, S. Shenker and L. Susskind, Phys. Rev. D55 (1997) 5112, hep-th/9610043.

9. N. Ohta and J.-G. Zhou, Nucl. Phys. B522 (1998) 125, hep-th/9801023.

10. M. L. Meana, M. A. R. Osorio and J. P. Peñalba, hep-th/9803058.

11. B. Sathiapalan, Mod. Phys. Lett. A13 (1998) 2085, hep-th/9805126.

12. M. B. Green, Nucl. Phys. B381 (1992) 201.

13. M. M. Vázquez-Mozo, Phys. Lett. B388 (1996) 494, hep-th/9607052.

14. M. B. Green, Phys. Lett. B266 (1991) 325. 